\title{
Humangeographie meets Geographiedidaktik - Interdisziplinäre Annäherungen an aktuelle Forschungsmethoden
}

\author{
*matthias.naumann@aau.at, Institut für Geographie und Regionalforschung, Universität Klagenfurt ( $\square$ korresp. Autor) \\ **anna.oberrauch@ph-tirol.ac.at, Institut für fachdidaktische und bildungswissenschaftliche Forschung und Entwicklung, Pädagogische \\ Hochschule Tirol \\ *** nicole.raschke@tu-dresden.de, Fachrichtung Geowissenschaften, Technische Universität Dresden
}

eingereicht am: 26.10.2021, akzeptiert am: 22.11.2021

\begin{abstract}
Das vorliegende Schwerpunktheft ist von der Idee einer interdisziplinären Auseinandersetzung mit neuen Methoden der Humangeographie getragen, in deren Rahmen eine gegenseitige Bereicherung fachwissenschaftlicher wie fachdidaktischer Perspektiven angestrebt wird. Autor*innen-Teams aus Fachwissenschaftler*innen und Fachdidaktiker*innen haben in kollaborativen Schreibprozessen eine aktuelle humangeographische Forschungsmethode bzw. -perspektive hinsichtlich ihres Potentials für geographische Forschung und den Geographieunterricht diskutiert. Im Anschluss an Trends in der aktuellen Entwicklung humangeographischer Methodik sowie an gegenwärtige Herausforderungen für die geographische Bildung, werden Hintergründe, Methode und Ergebnisse dieses Prozesses dargestellt und reflektiert.
\end{abstract}

Keywords: Interdisziplinarität, qualitative Methoden, humangeographische Forschung, geographische Bildung, kollaboratives Schreiben

\section{Human Geography meets Geographical Education - Interdisciplinary Approaches to Current Research Methods}

This special issue is based on the idea of an interdisciplinary dialogue on new methods in human geography. This dialogue could enrich the perspectives of Human Geography as well as of Geographical Education. In collaborative writing processes, teams of authors from Human Geography and Geographical Education discussed current human geography research methods with regard to its potential for geographical research and teaching. Following current trends in the development of human geography methodologies and challenges for geographical education, the background, method and results of this process of collaboration are presented and reflected.

Keywords: interdisciplinarity, qualitative methods, human geography research, education in geography, collaborative writing

\section{$1 \quad$ Einleitung}

"Geography is, what Geographers do" - dieser viel zitierte und auch kritisierte Satz von R. J. Johnston wirft nicht nur die Frage nach den Inhalten der Geographie auf, sondern auch nach dem ,How Geographers do, what Geographers do?" Das vorliegende Schwerpunktheft widmet sich dieser Frage anhand eines Austausches zwischen Humangeograph*innen und Geographiedidaktiker*innen, die sich auf einen kollaborativen Schreibprozess zum Thema einer ausgewählten humangeographischen Methode eingelassen haben. Dabei verfolgt das Heft das grundlegende Ziel, den Dialog zu Forschungsmethoden zwischen
Humangeographie und Geographiedidaktik zu vertiefen und $\mathrm{zu}$ weiterem Austausch anzuregen. Die Idee zum Heft entstand aus interdisziplinären Erfahrungen der Herausgeber*innen in Forschung und Lehre, bei denen die fachlichen Grenzen und Möglichkeiten für Kooperationen und Perspektiven immer wieder neu ausgelotet wurden. Gerade im Bereich der Forschungsmethoden erscheint die Berücksichtigung der Perspektiven der Humangeographie und der Geographiedidaktik fruchtbar, weil Methoden als Wege der Erkenntnisgewinnung eine zentrale Rolle in fachwissenschaftlicher wie auch pädagogischer Arbeit spielen. Damit ist das Heft selbst Ausdruck eines Prozesses, der, das zeigen die Einschätzungen der 
Autor*innen dieses Heftes, als gewinnbringend und informativ eingeschätzt wurde. Autor*innen-Tandems, bestehend aus Fachwissenschaftler*innen und Fachdidaktiker*innen wurden dazu angeregt, in einem kollaborativen Entwicklungs- und Schreibprozess eine neue humangeographische Forschungsmethode bzw. -perspektive in Bezug auf ihr Potential für Erkenntnisgenerierung in geographischer Forschung sowie im Geographieunterricht in den Blick zu nehmen ${ }^{1}$.

Im Aufeinanderbeziehen zwischen Humangeographie und Geographiedidaktik sind Synergien, aber auch Reibungspunkte und offene Fragen zu bestimmen. Damit soll nicht behauptet werden, dass dieses Aufeinanderbeziehen nicht bereits stattfindet. Fachwissenschaft und -didaktik sind durch vielfältige Wissensbeziehungen miteinander verbunden und arbeiten in wissenschaftlichen Netzwerken zusammen. In einer Untersuchung akademischer Wissensbeziehungen anhand von Publikations- und Zitationsverhalten stellen Steinbrink und Aufenvenne (2016) fest, dass es starke Zitationsbeziehungen - v.a. zwischen Fachdidaktik und Humangeographie - gibt, wobei diese Beziehungen aus der Didaktik in die Humangeographie hinein sehr viel stärker ausgeprägt sind als in die Gegenrichtung. Einen Schritt weiter zu gehen und Wissensbeziehungen mit dem Ziel kollaborativer Wissensproduktion auf Augenhöhe in der direkten Zusammenarbeit von Fachwissenschaftler*innen und Fachdidaktiker*innen anzuregen - das ist das Ziel dieses Schwerpunkthefts. Das Heft als Ganzes sowie jeder einzelne Beitrag für sich lassen sich als Produkt eines solchen Austauschprozesses lesen. Die einzelnen Beiträge stellen exemplarisch neuere Zugänge und Methoden der humangeographischen Forschung vor (partizipative Aktionsforschung, kritische Kartographie, Bildanalysen, Online-Fragebögen, Analyse von Twitter-Daten, Mobility Mapping und Walking Interviews) und diskutieren anhand konkreter Unterrichtsszenarien, wie diese in geographische Bildungsprozesse eingebunden werden können.

In diesem Einleitungsbeitrag werden zunächst einige Trends in der aktuellen Entwicklung humangeographischer Methoden sowie gegenwärtige Herausforderungen für die geographische Bildung vorgestellt, die den Anlass und Hintergrund für dieses Heft darstellen. Anschließend wird der Prozess des kollaborativen Schreibens als methodischer Zugang zum interdisziplinären Vorhaben diskutiert. Danach zeigt Kapitel 3 die Reflexion dieses kollaborativen Prozesses auf. Das Fazit formuliert einige vorläufige Thesen und wei-

\footnotetext{
In Bezug auf die Fachdidaktik ist also eine unterrichtspraktische Perspektive vorherrschend, d.h. der Dialog zielt auf die gemeinsame Erkundung des Potentials für (neue) methodische Herangehensweisen im G(W)-Unterricht ab, während das Feld fachdidaktischer Forschung (vorerst) unberücksichtigt bleibt.
}

tergehende Fragen für den künftigen Austausch zwischen Humangeographie und Geographiedidaktik.

\section{Humangeographische Methoden im Wandel und neue Herausforderungen der geographischen Bildung}

Die Humangeographie, ihre Paradigmen und ihre Wege zu neuen Erkenntnissen befinden sich im permanenten Wandel. Dies trifft auch auf die Geographiedidaktik und den Geographieunterricht zu. Dabei lassen sich einige gemeinsame Trends feststellen, die sowohl Humangeographie als auch Geographiedidaktik betreffen. Im Folgenden stellen wir Beispiele für den methodischen Wandel in der Humangeographie und aktuelle Herausforderungen für die geographische Bildung dar, bevor wir darlegen, wie eine kollaborative, interdisziplinäre methodische Auseinandersetzung von Humangeographie und Geographiedidaktik diese Herausforderungen aufgreifen kann.

\subsection{Wandel humangeographischer Methoden}

Die Frage, was eine genuine humangeographische Methodik ist, lässt sich nicht einfach beantworten. Zwar gilt die Kartierung als eine ,urgeographische' Methode, doch darüber hinaus bedient sich die Humangeographie methodischer Zugänge, die häufig ursprünglich aus anderen Disziplinen stammen. Hierzu zählen etwa ethnographische Methoden aus der Anthropologie, politikwissenschaftliche Netzwerkanalysen oder Gruppeninterviews, die zuerst in den Erziehungsund Gesundheitswissenschaften entwickelt wurden. Dennoch entwickelte sich in der internationalen wie auch in der deutschsprachigen Humangeographie eine umfangreiche Methodendiskussion (für einen deutschsprachigen Überblick siehe Wintzer 2018). Zu einzelnen in der Humangeographie, aber auch in der Geographiedidaktik verwendeten Methoden wie der Diskursanalyse (Glasze \& Mattissek 2021), dem Kritischen Kartieren (Dammann \& Michel 2022), visuellen Methoden (Schlottmann \& Miggelbrink 2015) oder Fokusgruppen (Gailing \& Naumann 2019) liegen methodologische Reflexionen vor. Insgesamt lassen sich einige aktuelle Trends feststellen, die den Wandel humangeographischer Methoden kennzeichnen.

Erstens umfassen digitale Geographien (für einen aktuellen Überblick siehe Bork-Hüffer et al. 2021) Möglichkeiten für neue methodische Zugänge. Digitale Technologien der Datenerhebung und Auswertung, zum Beispiel die Nutzung von Social-MediaDaten (siehe Beitrag Brendel et al. in diesem Heft), Online-Umfragen, aber auch Foto- und Videoaufnahmen mit Smartphones, erlauben die Generierung 
und Verarbeitung von großen Informationsvolumen, führen aber auch zu neuen forschungsethischen Fragen. Die Berücksichtigung von Mobilität in der Datenerhebung ist eng verknüpft mit neuen digitalen Technologien, die mobile Formen der Datenerhebung erlauben. Diese können in methodischen Zugängen des Mobility Mappings (siehe Beitrag Kordel et al. in diesem Heft) oder auch von Walking Interviews (siehe Beitrag Langner et al. in diesem Heft) zum Einsatz kommen. Weitere Stichworte für neue empirische Gegenstände, aber auch für methodische Innovationen in der Humangeographie wären Big Data, Digital Humanities oder auch virtuelle Realitäten.

Zweitens gewinnt die Reflexivität in humangeographischer Forschung zunehmend an Bedeutung (Meyer et al. 2018). Dies umfasst unter anderem die kritische Reflexion der Rolle von Forschenden und deren Positionalität, der Hierarchien zwischen Forschenden und Beforschten, aber auch einen transparenten und sensiblen Umgang mit Forschungsdaten. Neben der Entwicklung experimenteller Methoden, etwa in der aktivistischen Stadtforschung, erfahren auch etablierte Methoden, wie die Statistik (Kemper 2018), eine kritische Reflexion. Partizipative Methoden verfolgen das Ziel, die Rolle der Teilnehmenden an Forschungsprojekten gegenüber den Forschenden zu stärken und gesellschaftliche Veränderungen zu initiieren. Damit werden Ansätze der Partizipativen Aktionsforschung (siehe Beitrag Pettig et al. in diesem Heft) und des Kritischen Kartierens (siehe Beitrag Michel \& Gryl in diesem Heft) wichtiger.

Drittens wächst auch eine interdisziplinäre Orientierung humangeographischer Methoden. Hierfür stehen sozialwissenschaftliche Zeitschriften wie das ,International Journal of Qualitative Methods', ,Methodological Innovations' oder ,FQS - Forum Qualitative Sozialforschung', in denen auch unter der Beteiligung von Humangeograph*innen methodologische Debatten geführt werden. Am Beispiel der Verwendung ethnographischer Methoden zeigt sich, dass die Grenzen zwischen akademischen Disziplinen verschwimmen und bewusst überschritten werden. Das Ausloten und bewusste Verwischen der Grenzen zwischen wissenschaftlichen Disziplinen am Beispiel von Humangeographie und Geographiedidaktik wird auch mit Blick auf die gegenwärtigen Herausforderungen geographischer Bildung deutlich, die parallel zu den dargestellten Trends humangeographischer Forschungsmethoden anhand dreier Themenfelder dargestellt werden.

\subsection{Aktuelle Herausforderungen geographischer Bildung}

In fachdidaktischen und bildungspolitischen Auseinandersetzungen zu geographischer Bildung lassen sich zentrale Themenfelder ausmachen, die den Diskurs hinsichtlich geeigneter methodischer Zugänge zur Gestaltung von Lerngelegenheiten entscheidend prägen. Die gegenwärtigen Herausforderungen geographischer Bildung stehen in einem engen Zusammenhang zu gesamtgesellschaftlichen Veränderungen wie etwa der ökologischen Krise und damit verbundenen Fragen nach sozialer und ökologischer Gerechtigkeit. Geographischer Bildung kommt die Verantwortung $\mathrm{zu}$, sich in diesem Kontext kritisch zu reflektieren und zu positionieren, die eigenen Möglichkeiten zu prüfen und geographisches Lernen zukunftsfähig zu gestalten, ohne dabei in eine zweckrationale Logik der Krisenbewältigung durch Bildung zu verfallen (Hamborg 2020). Im folgenden Abschnitt geht es exemplarisch um drei Herausforderungen geographischer Bildung: Partizipation und Bildung für nachhaltige Entwicklung (BNE), Digitalisierung und Reflexivität sowie fächerübergreifendes und außerschulisches Lernen.

Geographie als Leitfach einer BNE zu verstehen, speist sich wesentlich aus dem integrativen und systemorientierten Charakter des Faches sowie dem fachpolitischen Bildungsauftrag im Hinblick auf fachspezifische Kompetenzen (DGfG 2020). Vor dem Hintergrund der Kritik an einer instrumentellen Auslegung von BNE und der Etablierung eines kritischemanzipatorischen Bildungsverständnisses im Kontext einer nachhaltigen Entwicklung (Pettig 2021), stehen Fragen nach den Möglichkeiten und Grenzen einer echten Partizipation von Lernenden in geographischen Bildungsanlässen im Raum. Akteur*innen aus Bildungskontexten sind zur Teilhabe an gesellschaftlichen Transformationsprozessen aufgefordert und angehalten, lebensweltliche Bezüge zu stärken, interdisziplinäre, handlungs- und projektorientierte Lerngelegenheiten zu gestalten sowie Möglichkeiten zur Teilhabe zu schaffen. Das Verhältnis von BNE und geographischem Lernen sowie die Möglichkeiten und Grenzen der Umsetzung im (Fach-)Unterricht sind zentrale Herausforderungen, die Fragen nach der Aktualisierung postkolonialer Macht- und Wissensregime, nach der Funktionalisierung von Bildung im Kontext einer BNE (Hamborg 2017) oder auch dem Umgang mit Normativität in Bildungsprozessen (Thomsen 2020) einschließen. Diese Aushandlungen sind auch geographiedidaktisch und unterrichtspraktisch relevant. Neben einem kritisch-emanzipatorischem Verständnis transformativen Lernens sind pädagogische Ansätze, die Erleben, Erfahrung und deren Reflexion in den Fokus rücken, etwa experiential learning (Dewey 2002; Kolb 1984), bedeutsam und müssen bei der Auswahl und Ausgestaltung spezifischer Methoden des Lernens berücksichtigt werden.

Damit eng verbunden sind zweitens die sich verändernden Verhältnisse durch Digitalität, Digitalisie- 
rung oder Digitalismus (Rhode-Jüchtern 2020). In einer Kultur der Digitalität (Stalder 2016) sind Prozesse der Raumaneignung und Raumproduktion sowie Erkenntnis- und Lernprozesse digital durchdrungen. Während mit Digitalisierung weitere Möglichkeiten der Partizipation, Kreativität und Kollaboration verbunden sein können, besteht gleichzeitig die Herausforderung des adäquaten Umgangs mit Algorithmizität und Kontrolle. Aus dem ambivalenten Verhältnis zwischen Abhängigkeit von und Autonomie durch digitale Praktiken geht ein Bildungsanspruch hervor, der Menschen befähigen soll, sich in einer digital durchdrungenen Gesellschaft zu orientieren, gesellschaftliche Verhältnisse in der digitalen Welt kritisch-reflexiv zu hinterfragen und diese zu gestalten. Geographische Bildung ist im Hinblick auf ihren Bildungsgehalt, die Änderungen in den Bezugswissenschaften sowie den Prozess des Lernens inhaltlich und methodisch digital geprägt. Digitale Technologien konfrontieren Schule und Unterrichtspraxis mit neuen Herausforderungen, die nicht nur technisch sind, sondern eben auch Inhalte betreffen (Felgenhauer $\&$ Gäbler 2019). Ähnlich wie in humangeographischen Kontexten bestehen in einer Kultur der Digitalität neue Möglichkeiten der Initiierung von Lernanlässen, aber auch ein neuer Anspruch an geographische Bildung selbst. Im Zusammenhang mit transformativem Lernen in einer Kultur der Digitalität kommt den reflexiven Bildungsprozessen eine besondere Bedeutung zu, weil sie Schüler*innen ermöglichen, eigene Entscheidungen zu hinterfragen, mündig zu urteilen und autonom zu handeln. Dies wird in den Ansätzen zu Spatial Citizenship Education umfassend betrachtet (Jekel et al. 2015).

Eine Möglichkeit zur konstruktiven Wendung dieser Herausforderungen stellt eine Öffnung der Institution Schule zur Lern- und Bildungslandschaft dar. Diese ermöglicht das Durchbrechen bestehender unterrichtlicher Routinen und eröffnet neue Lernwege sowie pädagogische Angebote (Karpa et al. 2015). Weiterhin gilt eine damit verbundene Lebensweltorientierung in unterrichtlichen Settings als Chance, weil in authentischen Kontexten konkrete Problem- bzw. Fragestellungen aufgeworfen werden, die für Lernende relevant sind (Pospiech et al. 2020). Im Rahmen eines regionalen Lernens (Diersen \& Paschold 2020) werden Wahrnehmung und Kenntnis gesellschaftlicher Partizipationsmöglichkeiten durch Kooperationen mit außerschulischen Lernorten gefördert. Insofern ist außerschulisches Lernen auch anschlussfähig an nachhaltigkeits- und digitalisierungsbezogene Debatten gesellschaftlicher Transformation. Außerschulische Lernorte bieten vielfältige Zugänge zu fächerübergreifenden Lerninhalten, indem in realweltlichen Kontexten der Lernenden konkrete Problem- bzw.
Fragestellungen aufgeworfen und mit problem-, projekt- und forschend-entdeckenden Zugängen bearbeitet werden (Brundiers \& Wiek 2011).

Ein intensivierter Dialog zwischen Humangeographie und Geographiedidaktik bietet die Möglichkeit, die Parallelen gegenwärtiger Trends und damit verbundener Herausforderungen zwischen den Disziplinen auszuloten und anhand ausgewählter innovativer Methoden einen Beitrag zur Bereicherung beider Disziplinen zu leisten. Dabei wird der Begriff "Methode" sehr breit ausgelegt, indem sowohl paradigmatische Formate (bspw. Partizipative Aktionsforschung) als auch ganz konkrete Instrumente der Erkenntnisgewinnung (bspw. Online-Umfragen) in den Blick genommen werden. Das hier zugrundeliegende Methodenverständnis wendet sich gegen einen formalistischen Methodenbegriff, der in der Logik einer nachgeordneten „Erfüllungsdidaktik“ humangeographische Methoden als algorithmische Instrumente für den Unterrichtsprozess formuliert. Das Themenheft hinterfragt die Bedingungen für die Auswahl, den Einsatz, die Durchführung und die Reflexion methodischer Entscheidungen im Lernprozess und will Methoden nicht isoliert, sondern im Zusammenwirken mit weiteren bildungs- und forschungsbezogenen Kontexten diskutieren. Der Prozess des kollaborativen Schreibens wird hierfür als geeigneter Rahmen für diesen Austausch erachtet.

\subsection{Kollaboratives Schreiben als methodischer Rahmen für den Austausch zwischen Hu- mangeographie und Geographiedidaktik}

Den methodischen Rahmen für die Entwicklung der Heftbeiträge zu humangeographischen Forschungsmethoden und ihren Potentialen für die Umsetzung im G(W)-Unterricht bildet das kollaborative Schreiben. In diesem Abschnitt soll dieser Rahmen kurz ausgeführt werden, da er einen wichtigen Reflexionshintergrund für die Erfahrungen der Teilnehmenden bildet.

Das kollaborative Schreiben kann als eine Methode dialogischer Wissensproduktion verstanden werden. Kollaboration kennzeichnet im Allgemeinen ein koordiniertes, synchrones Bemühen, um gemeinschaftlich an der Lösung eines Problems oder der Antwort auf eine Frage zu arbeiten (Roschelle \& Teasley 1995). Im Unterschied zur Kooperation, bei der eine Aufgabe unter dem Aspekt der Optimierung unter den Beteiligten aufgeteilt wird, setzt Kollaboration stärker auf die interaktive Aushandlung, Ko-Konstruktion und die Hervorbringung eines gemeinsamen Ergebnisses, das die einzelnen Teilnehmenden allein nicht hätten erreichen können (Lehnen 2017; Camarihna-Matos \& Afsarmanesh 2005; Roschelle \& Teasley 1995). 
Beim kollaborativen Schreiben zielt der Prozess der Zusammenarbeit auf eine kollaborative Textproduktion ab, wobei unzählige Formen, Techniken und Definitionen unterschieden werden können. Lowry et al. (2004: 72) definieren kollaboratives Schreiben als "iterative and social process that involves a team focused on a common objective that negotiates, coordinates, and communicates during the creation of a common document".

Das eigentliche Schreiben macht dabei nur einen Teilaspekt der Zusammenarbeit aus. Jede Aktivität die zum Abschlussdokument einen Beitrag leistet, wie etwa Brainstorming und Ideenfindung, Recherche und Forschung, Planung und Organisation, Überarbeitung und Korrektur, werden als zum Schreibprozess zugehörig betrachtet (Rice \& Huguley 1994; Lowry et al. 2004). Der Prozess reicht somit von der Planung bis zur Publikation des Produktes und wird mündlich oder schriftlich durch konversationelle Schreibinteraktionen (Lehnen 2017) begleitet. Diese werden als Ko-Konstruktionen begriffen, wenn auch nicht alles in der Interaktion gemeinschaftlich hervorgebracht wird (Kostouli 2005, zit. n. Lehnen 2017). Die interaktive Aushandlungssituation soll dabei „unterschiedliche Perspektiven auf den Schreib- bzw. Argumentationsgegenstand" provozieren (Lehnen 2017: 307). Diese werden sichtbar, für die Textproduktion verfügbar, und sie fordern die stetige Reflexion eigener Perspektiven und die Entwicklung eines Konsenses heraus (Lehnen 2017; Lowry et al. 2004). Dies basiert auf dem Verständnis, dass Kollaboration stets partizipatorisch und dialogisch gestaltet ist und im Gegensatz zu bürokratischen und hierarchisierten Formen der Kollaboration, frei von Hierarchien oder zu starken Formalismen ist (Krishnan et al. 2019; Ede \& Lunsford 1992).

Auch wenn kollaboratives Schreiben eine Gruppenaufgabe ist, werden einzelne Teile und Aufgaben oft verteilt und individuell erarbeitet (Lowry et al. 2004). Das Schreibvorhaben kann dabei in unterschiedlichen Konstellationen organisiert werden, die alle mit verschiedenen Vor- und Nachteilen einhergehen (Cap et al. 2012). Beim „sequenziellen Schreiben“ schreiben die Teammitglieder nacheinander schrittweise am Text weiter, während beim „redaktionellen Schreiben“, nach einer gemeinsamen Planungsphase, Textteile einzeln verfasst und von einem*einer Redakteur*in zusammengefügt werden. Weitere Formen stellen das „parallele Schreiben“, bei dem Teilnehmende stärker unterschiedliche Rollen im gemeinsam organisierten Prozess übernehmen (z. B. Visualisieren, Recherchieren, Formulieren) oder das „reagierende Schreiben“, bei der die Autor*innen gleichzeitig am Text schreiben und dabei stark in Interaktion und Aushandlung treten, dar. Es zeigt sich, dass kollaboratives Schreiben ein komplexer Prozess ist, bei dem vor allem der Umgang mit auftretenden Inkonsistenzen, unterschiedlichen Sichtweisen, Zielabsichten und Normen, die oft mit einer unzureichend geklärten Diskurszugehörigkeit zusammenhängen, zu Problemen führen kann (Cap et al. 2012). Ebenso werden ungleichmäßig verteilte Beiträge der Autor*innen in allen Phasen des Schreibprozesses, die Organisation und nicht-hierarchische Koordination des Prozesses und die notwendige Offenheit aller Partizipierenden aufgrund mangelnder Planbarkeiten des nicht-linearen und dynamischen Prozesses als mögliche Herausforderungen identifiziert (Lowry et al. 2004).

Die konzeptionellen Hintergründe zur Methode des kollaborativen Schreibens, mit all ihren unterschiedlichen Auslegungen und Techniken, ließen diese Zugangsweise als sinnvolle methodische Rahmung für das Publikationsprojekt erscheinen. Welche Varianten, Wege der Planung, Prozesse der Organisation und Aushandlung die Autor*innen-Teams zum Verfassen ihrer Beiträge wählten bzw. sich im Zuge des Tuns herauskristallisierten, war ihnen dabei selbst überlassen. Die Schreibaufgabe und die Integration unterschiedlicher fachlicher Expertisen und Perspektiven der Autor*innen-Teams (fachwissenschaftliche, forschungsmethodische, fachdidaktische sowie unterrichtspraktische) machten mit Sicherheit einen großen Bedarf an Interaktion und gemeinsamer Konzeptionalisierung nötig.

Bevor der Prozess und dessen Ergebnisse reflektiert werden, sollen zunächst die Rahmenbedingungen für den kollaborativen Schreibprozess erläutert werden. Die Einladung der Autor*innenTandems erfolgte durch die Herausgeber*innen für eine Forschungsmethode, die zur Expertise der angesprochenen Fachwissenschaftler*innen und Fachdidaktiker*innen gehört. Die Autor*innenTandems zogen in einigen Fällen weitere Fachwissenschaftler*innen, Fachdidaktiker*innen oder Unterrichtspraktiker*innen aus ihren Netzwerken hinzu. Im Rahmen des Call for Papers für das Heft wurde eine mögliche Grobgliederung des Beitrags vorgeschlagen, die sich auf die Darstellung der Methode aus einer humangeographischen Perspektive und fachdidaktischer Wendung bezog. Dabei sollte die Diskussion beider Perspektiven anhand eines konkreten Unterrichtskonzeptes bzw. Anwendungsbeispiels der Methode erfolgen, bevor aus fachdidaktischer und humangeographischer Sicht ein Fazit zu ziehen war. Die Beiträge durchliefen anschließend ein Double-Blind-Peer-Review-Verfahren, wobei auch hier die Interdisziplinarität ausschlaggebend war. So wurde darauf geachtet, dass die Gutachter*innen sowohl die fachwissenschaftliche als auch die fachdidaktische Perspektive einzubringen in der Lage sind. Die für 
die Zeitschrift bewährten Textkategorien (Fachwissenschaft, Fachdidaktik und Unterrichtspraxis) wurden dabei durch die Kombination von wissenschaftlichem und anwendungsorientiertem Beitrag sowie durch die Vermischung der Disziplinen bewusst durchbrochen.

\section{Interdisziplinäre Reflexionen zum gemeinsamen Schreibprozess}

Auf der \#GeoWoche2021 im Oktober 2021 wurde im Rahmen einer Fachsitzung dem Autor*innenKollektiv des Heftes die Möglichkeit gegeben, ihre (Zwischen-)Ergebnisse des Publikationsprojektes zu präsentieren und im gemeinsamen Austausch den kollaborativen Entwicklungs- und Schreibprozess zu reflektieren. In Kurzstatements und Diskussionsbeiträgen wurde erörtert, wie sich die interdisziplinäre Zusammenarbeit zwischen Humangeographie und Geographiedidaktik gestaltete, welche Potentiale und Herausforderungen sich dabei ergaben und welche Schlussfolgerungen sich daraus für beide Disziplinen und ihre zukünftige Zusammenarbeit ziehen lassen. Im Folgenden werden die Ergebnisse der Session kurz zusammengefasst. Da nur ein Teil der Autor*innen an der Fachsitzung teilnahm, können nicht alle Perspektiven von den Beitragenden zum Heft wiedergegeben werden.

Die Tatsache, dass die Autor*innen teilweise einander unbekannt waren, war eine Herausforderung, die jedoch als sehr gewinnbringend bewertet wurde. Das gegenseitige Kennenlernen und Annähern im gemeinsamen Schreibprozess und das Einlassen auf die jeweils andere Person und deren Perspektive wurde durchwegs als Bereicherung aufgefasst, auch wenn die Covid-19-Pandemie ein persönliches Kennenlernen erschwerte.

Für den Prozess selbst lassen sich unterschiedliche Herangehensweisen feststellen: Einige Autor*innen begannen nach einer gemeinsamen Planungsphase zunächst parallel an ihren Kapiteln zu schreiben (vgl. Variante des „redaktionellen Schreibens"). Dies wurde v. a. aus der Perspektive der Fachdidaktik als herausfordernd empfunden, da der fachwissenschaftliche Teil als wichtige Voraussetzung für die Weiterentwicklung des eigenen fachdidaktischen Teils eingeschätzt wurde. Insofern ist der gemeinsame Beitrag dann schrittweise durch intensive Interaktion zusammengewachsen. Andere Autor*innengruppen verwarfen bei einem ersten Meeting sehr schnell die Idee, einzelne Kapitel entsprechend der disziplinären Schwerpunkte untereinander aufzuteilen, um der Gefahr einer bloßen Übertragung und vereinfachenden Reduktion fachwissenschaftlicher systematischer Prozesse in bzw. für die Unterrichtspraxis, zu entgehen. Der Prozess gestaltete sich anschließend herausfordernder, aber auch spannender, denn im Aufeinanderzugehen wurde der gesamte Text gemeinsam geschrieben (vgl. Modus des ,reagierenden Schreibens“).

Mit Blick auf die Wahrnehmung des kollaborativen Schreibprozesses lässt sich feststellen, dass ein erster Schritt darin bestand, darüber Einigkeit zu schaffen, dass es um keine „Abbilddidaktik“ im Sinne einer reinen Übersetzung fachwissenschaftlicher Methoden in die Unterrichtspraxis gehen kann, sondern um einen gemeinsamen, integrativen und produktiven Prozess. Der Schreibprozess wurde als herausfordernd beschrieben, insbesondere mit Blick auf die Klärung von Begriffsverständnissen, theoretischen Perspektiven und dem Abstraktionsgrad. Dabei entstand ein besonderes Spannungsfeld darin, die fachwissenschaftliche Weite des Themas im Blick zu haben und gleichzeitig auf einer konkreten Umsetzungsebene im Schulunterricht zu argumentieren.

Aus fachwissenschaftlicher Perspektive wurde der Austausch von den Beteiligten als sehr fruchtbar und vielversprechend empfunden, was sich auch darin zeigt, dass einige Autor*innen-Teams zukünftig weitere gemeinsame Publikationsprojekte angehen wollen. Übereinstimmend stellen die an der Podiumsdiskussion beteiligten Autor*innen fest, dass sich der gemeinsame Fokus, der sich aus der Modifizierung des vorgegebenen Themas erst entwickelte, sowie das gemeinsame Format des Beitrages in Anpassung der vorgegebenen Struktur erst im Laufe des Arbeitsprozesses entwickelten.

Für die Humangeographie eröffneten sich neue Perspektiven, die beispielsweise darin bestehen, Inspirationen aus der partizipativen Aktionsforschung für Arbeiten im Bereich der Geographien der Kindheit und Jugend zu nutzen. Zahlreiche gegenseitige Impulse wurden erkannt, wobei die Anleihen, die von der Geographiedidaktik bzw. der Humangeographie in ihren jeweiligen benachbarten Bezugsdisziplinen - die sich teilweise überschneiden, aber auch unterscheiden - gemacht werden, auch für die jeweils andere Disziplin neue Perspektiven erbringen können. Ein interessantes zukünftiges Handlungsfeld für eine fruchtbare interdisziplinäre Vernetzung bieten Stadtgeographie und Geographiedidaktik in Bezug auf die Gestaltung von Reallaboren als transdisziplinäre Forschungs- und Entwicklungsräume im Zuge gesellschaftlicher Transformation. Die fachdidaktische Perspektive liefert hierfür zahlreiche Impulse und Erfahrungen, z. B. hinsichtlich der Einbindung von Akteur*innen in partizipativen Forschungsprozesse auf Augenhöhe, die Berücksichtigung von Konzepten und Perspektiven aller Beteiligten und die Gestaltung offener Diskussions- und Reflexionsräume. 
Im Rahmen der Reflexion auf der \#GeoWoche2021 wurden Lernprozesse in beide Richtungen angesprochen - was kann die Fachwissenschaft von der Fachdidaktik lernen und was kann die Fachdidaktik von der Fachwissenschaft lernen-, wobei sich letztere häufig als die dominantere Sichtweise niederschlägt, die methodische Diskussionen weiterhin maßgeblich bestimmt. Ebenso wurde aber die Einschätzung geäußert, dass es weniger um die Frage gehen kann, was die Disziplinen jeweils voneinander lernen können, sondern vielmehr um die Wertschätzung der unmittelbaren Erfahrung, dass das Ergebnis des gemeinsamen Arbeitsprozesses durch die dialogische Entwicklung auf Augenhöhe gewachsen und qualitativ überzeugend ist. In diesem Kontext wurde auch die Frage aufgeworfen, ob disziplinäre Grenzen mittlerweile nicht obsolet geworden sind. So gibt es viele Beispiele für die fruchtbare $\mathrm{Zu}-$ sammenarbeit zwischen Humangeographie und Geographiedidaktik, z. B. das "Netzwerk Visuelle Geographien "2 oder der Arbeitskreis "Qualitative Methoden"3 in der Deutschen Gesellschaft für Geographie. Die Herausforderungen bestehen, so die Einschätzungen in der Fachsitzung, eher im kollaborativen Schreibprozess selbst, dem sich Zusammenfinden in Bezug auf das gemeinsame Ziel, die gemeinsamen Begriffe, theoretischen Perspektiven etc., anstatt in der Überwindung disziplinärer Grenzen.

Abschließend soll diskutiert werden, welche Schlussfolgerungen aus diesem Publikationsprojekt für die weitere Zusammenarbeit von Humangeographie und Geographiedidaktik gezogen werden können.

\section{$4 \quad$ Fazit}

Für die Humangeographie wie auch für die Geographiedidaktik stellen sich drängende Herausforderungen, die auch die methodischen Zugänge von Forschung und Lehre betreffen. Beispielsweise ist anzunehmen, dass die Auswirkungen von Covid-19 in Forschung, Lehre und Unterricht bleibende Spuren hinterlassen werden. Die Klimakrise macht den Bedarf für eine integrativ arbeitende Geographie immer stärker deutlich, wie auch die Digitalisierung die Vernetzung zwischen verschiedenen Disziplinen und deren Internationalisierung weiter befördern wird. Eine gemeinsame Reflexion von Humangeographie und Geographiedidaktik kann methodische Zugänge zu den genannten Herausforderungen hinterfragen und weiterentwickeln. Dafür können auch die disziplin-

\footnotetext{
https://visqual.leibniz-ifl-projekte.de/

3 https://www.qualitative-methoden.giub.unibe.ch/qualitativemethoden/
}

spezifischen Unterschiede einen Anlass bieten. Diese zeigen sich beispielsweise in einer unterschiedlichen Ausrichtung der wissenschaftlichen Theoriebildung oder auch bei der Dissemination von Forschungsergebnissen. So nehmen in einer fachdidaktischen Perspektive der Transfer in die gesellschaftliche Breite und die Praxis geographischer Bildung einen hohen Stellenwert ein. Die Unterschiede sind nicht unüberbrückbar, wie die Debatte um eine „Angewandte Kritische Geographie" (Kuge et al. 2020) zeigt. Eine Diskussion über hierfür geeignete methodische Zugänge steht aber noch am Anfang.

Die Bearbeitung der aufgezeigten Herausforderungen könnte zu gemeinsamen Forschungsvorhaben, Lehrveranstaltungen, schulischen Projekten oder auch weiteren gemeinsamen Publikationsprojekten führen. Denkbar wären hier die Vertiefung und Erweiterung der Diskussion um einzelne Forschungsmethoden oder methodische Zugänge, die Reflexion des forschenden Lernens an Universitäten und Schulen oder auch die didaktische Vermittlung sowie der Anwendungsbezug kritischer Gesellschaftstheorie.

Die positiven Erfahrungen mit dem „Experiment“ dieses Schwerpunktheftes lassen darauf hoffen, dass sich künftige kollaborative Projekte von Humangeographie und Geographiedidaktik - den Worten eines Autors auf der \#GeoWoche2021 folgend - ,auch ohne Notwendigkeit eines externen Triggers" in Form eines Call for Papers, sondern noch stärker aus einem kontinuierlichen, alltäglichen Austausch heraus ergeben könnten.

\section{Danksagung}

Wir danken allen Autor*innen des Heftes, für das sich Einlassen auf den Prozess und das Mitgestalten des nun vorliegenden Publikationsprojekts. Ebenso gilt ein besonderer Dank allen Teilnehmer*innen der Fachsitzung auf der \#GeoWoche2021, deren Rückmeldungen und Perspektiven Eingang in diesen Artikel finden konnten: Nina Brendel, Andreas Eberth, Eva Nöthen, Fabian Pettig, Laurenz Virchow und Robert Vogler. Zudem bedanken wir uns für die Förderung der Publikation durch den Open Access Publikationsfonds der Universität Klagenfurt.

\section{Literatur}

Bork-Hüffer, T., H. Füller \& T. Straube (Hrsg.) (2021): Handbuch Digitale Geographien. Welt - Wissen Werkzeuge. Brill Schöningh, Paderborn.

Brundiers, K. \& A. Wiek (2011): Educating Students in Real-world Sustainability Research: Vision and Implementation. In: Innovative Higher Education 36(2). S. 107-124. DOI: 10.1007/s10755-010-9161-9 
Ede, L. \& A. Lunsford (1992): Singular Texts/Plural Authors. Perspectives on Collaborative Writing. Southern Illinois University Press, Carbondale.

Camarihna-Matos, L. M. \& H. Afsarmanesh (2008): Concept of Collaboration. In: Putnik, G. D. \& M. M. Cunha (Hrsg.): Encyclopedia of Networked and Virtual Organizations. Information Science Reference, Hershey, New York. S. 311-315.

Cap, C. H., W. Sucharowski \& W. Wendt (2012): Kollaboratives Schreiben von Texten im Web. In: HMD Praxis der Wirtschaftsinformatik 287. S. 61-68. DOI: 10.1007/BF03340738

Dammann, F. \& B. Michel (Hrsg.) (2022): Handbuch Kritisches Kartieren. transcript, Bielefeld.

Deutsche Gesellschaft für Geographie (DGfG) (2020): Bildungsstandards im Fach Geographie für den Mittleren Schulabschluss mit Aufgabenbeispielen. 10. aktual. Aufl., Bonn. https://geographie.de/wp-content/uploads/2020/09/Bildungsstandards_Geographie_2020_ Web.pdf (15.10.2021).

Dewey, J. (2002): Erfahrung und Erziehung. In: Horlacher, R. \& J. Oelkers (Hrsg.): Pädagogische Aufsätze und Abhandlungen (1900-1944). Pestalozzianum, Zürich. S. 227-283.

Diersen, G. \& L. Paschold (2020): Außerschulisches Lernen - ein Beitrag zur Bildung für nachhaltige Entwicklung und Inklusion. In: ZEP - Zeitschrift für internationale Bildungsforschung und Entwicklungspädagogik 43(1). S. 11-19. DOI: 10.31244/zep.2020.01.03

Felgenhauer, T. \& K. Gäbler (2019): Geographien digitaler Alltagskultur. Überlegungen zur Digitalisierung in Schule und Unterricht. In: GW-Unterricht 154. S. 5-20. DOI: 10.1553/gw-unterricht154s5

Gailing, L. \& M. Naumann (2019): Effizient und partizipativ? Fokusgruppen am Beispiel der geographischen Energieforschung. In: Geographische Zeitschrift 107(2). S. 107-129. DOI: $10.25162 / \mathrm{gz}-2019-0006$

Glasze, G. \& A. Mattissek (Hrsg.) (2021): Handbuch Diskurs und Raum. Theorien und Methoden für die Humangeographie sowie die sozial- und kulturwissenschaftliche Raumforschung. 3., überarb. und erw. Aufl. transcript, Bielefeld.

Hamborg, S. (2017): ,Wo Licht ist, ist auch Schatten ' Kritische Perspektiven auf Bildung für nachhaltige Entwicklung und die BNE-Forschung im deutschsprachigen Raum. In: Brodowski, M. (Hg.): Bildung für nachhaltige Entwicklung. Interdisziplinäre Perspektiven. Logos, Berlin. S. 15-31.

Hamborg, S. (2020): Bildung in der Krise. Eine Kritik krisendiagnostischer Bildungsentwürfe am Beispiel von ,Bildung für nachhaltige Entwicklung'. In: Kminek, H., F. Bank \& L. Fuchs (Hrsg.): Kontroverses Miteinander. Interdisziplinäre und kontroverse Positionen zur Bildung für eine nachhaltige Entwicklung (Frankfurter Beiträge für Erziehungswissenschaft). Goethe-Universität, Frankfurt am Main. S. 169-184.
Jekel, T., I. Gryl \& A. Oberrauch (2015): Education for Spatial Citizenship: Versuch einer Einordnung. In: GWUnterricht 137. S. 3-13.

Karpa, D., G. Lübbeke \& B. Adam (Hrsg.) (2015): Außerschulische Lernorte. Theorie, Praxis und Erforschung außerschulischer Lerngelegenheiten. Prolog-Verlag, Immenhausen bei Kassel.

Kemper, J. (2018): Die Stadt in der Statistik. In: Belina, B., M. Naumann \& A. Strüver (Hrsg.): Handbuch Kritische Stadtgeographie. Westfälisches Dampfboot, Münster. S. 113-119.

Kolb, D. A. (1984): Experiential Learning: Experience as the Source of Learning and Development. Englewood Cliffs, Prentice Hall.

Kostouli, T. (2005): Co-constructing writing contexts in classrooms. Scaffolding, collaboration, and symmetries of knowledge. In: Kostouli, T. (Hg.): Writing in Context(s): Textual Practices and Learning Processes in Sociocultural Settings. Springer, New York. S. 93-116.

Krishnan, J., S. Yim, A. Wolters \& A. Cusimano (2019): Supporting Online Synchronous Collaborative Writing in the Secondary Classroom. In: Journal of Adolescent \& Adult Literacy 63(2). S. 135-145. DOI: 10.1002/ jaal.969

Kuge, J., M. Naumann, H. Nuissl \& S. Schipper (2020): Angewandte und Kritische Geographie. Gemeinsame Herausforderungen, gemeinsame Perspektiven? In: Standort 44(4). S. 219-225. DOI: 10.1007/s00548020-00662-x

Lehnen, K. (2017): Kooperatives Schreiben. In: BeckerMrotzek, M., J. Grabowski \& T. Steinhoff (Hrsg.): Forschungshandbuch empirische Schreibdidaktik. Waxmann, Münster, New York. S. 299-314.

Lowry, P. B., A. Curtis \& M. R. Lowry (2004): Building a taxonomy and nomenclature of collaborative writing to improve interdisciplinary research and practice. In: Journal of Business Communication 41(1). S. 66-99. DOI: 10.1177\%2F0021943603259363

Meyer, F., J. Miggelbrink \& K. Beurskens (Hrsg.)(2018): Ins Feld und zurück - Praktische Probleme qualitativer Forschung in der Sozialgeographie. Springer Spektrum, Heidelberg.

Pettig, F. (2021): Transformative Lernangebote kritischreflexiv gestalten. Fachdidaktische Orientierungen einer emanzipatorischen BNE. In: GW-Unterricht 162. S. 5-17. DOI: 10.1553/gw-unterricht162s5

Pospiech, G., M. Niethammer, D. Wieser \& F.-M. Kuhlemann (2020): Begegnungen mit der Wirklichkeit. Chancen für fächerübergreifendes Lernen an außerschulischen Lernorten. hep Verlag, Bern.

Rhode-Jüchtern, T. (2020): Digitalisierung, Digitalität, Digitalismus. In: Zeitschrift für Didaktik der Gesellschaftswissenschaften 11(1). S. 9-22.

Rice, R. P. \& J. T. Huguley (1994): Describing collaborative forms: a profile of the team-writing process. In: Transactions on Professional Communication 37(3). S. 163-170. DOI: 10.1109/47.317482 
Roschelle, J. \& S. D. Teasley (1995): The Construction of Shared Knowledge in Collaborative Problem Solving. In: O’Malley, C. (Hg.): Computer Supported Collaborative Learning. Springer, Berlin/Heidelberg. S. 69-97.

Schlottmann, A. \& J. Miggelbrink (Hrsg.) (2015): Visuelle Geographien. Zur Produktion, Aneignung und Vermittlung von RaumBildern. transcript, Bielefeld.

Stalder, F. (2016): Kultur der Digitalität. 3. Aufl. Suhrkamp, Berlin.
Steinbrink, M. \& P. Aufenvenne (2016): Integrative Geographiedidaktik? Versuch einer Positionsbestimmung der Fachdidaktik innerhalb der deutschsprachigen Geographie. In: GW-Unterricht 142/143. S. 5-17. DOI: 10.1553/gw-unterricht142/143s5

Thomsen, S. (2020): Bildung in Protestbewegungen. Springer VS, Wiesbaden.

Wintzer, J. (Hg.) (2018): Sozialraum erforschen: Qualitative Methoden in der Geographie. Springer Spektrum, Heidelberg. 